\title{
Suppression of Poxvirus Replication by Resveratrol
}

\author{
Shuai Cao', Susan Realegeno' ${ }^{2}$, Anil Pant ${ }^{1}$, Panayampalli S. Satheshkumar ${ }^{2}$ and \\ Zhilong Yang ${ }^{1 *}$
}

${ }^{1}$ Division of Biology, Kansas State University, Manhattan, KS, United States, ${ }^{2}$ Poxvirus and Rabies Branch, Division of High-Consequence Pathogens and Pathology, National Center for Emerging and Zoonotic Infectious Diseases, Centers for Disease Control and Prevention, Atlanta, GA, United States

\section{OPEN ACCESS}

Edited by:

Jonatas Abrahao,

Universidade Federal de Minas

Gerais, Brazil

Reviewed by:

Danilo Oliveira,

Universidade Federal dos Vales do Jequitinhonha e Mucuri, Brazil lara Apolinario Borges, Universidade Federal de Minas

Gerais, Brazi

${ }^{*}$ Correspondence: Zhilong Yang zyang@ksu.edu

Specialty section: This article was submitted to Virology,

a section of the journal

Frontiers in Microbiology

Received: 31 August 2017 Accepted: 26 October 2017 Published: 17 November 2017

Citation:

Cao S, Realegeno S, Pant A, Satheshkumar PS and Yang Z (2017) Suppression of Poxvirus Replication by Resveratrol.

Front. Microbiol. 8:2196. doi: 10.3389/fmicb.2017.02196
Poxviruses continue to cause serious diseases even after eradication of the historically deadly infectious human disease, smallpox. Poxviruses are currently being developed as vaccine vectors and cancer therapeutic agents. Resveratrol is a natural polyphenol stilbenoid found in plants that has been shown to inhibit or enhance replication of a number of viruses, but the effect of resveratrol on poxvirus replication is unknown. In the present study, we found that resveratrol dramatically suppressed the replication of vaccinia virus (VACV), the prototypic member of poxviruses, in various cell types. Resveratrol also significantly reduced the replication of monkeypox virus, a zoonotic virus that is endemic in Western and Central Africa and causes human mortality. The inhibitory effect of resveratrol on poxviruses is independent of VACV N1 protein, a potential resveratrol binding target. Further experiments demonstrated that resveratrol had little effect on VACV early gene expression, while it suppressed VACV DNA synthesis, and subsequently post-replicative gene expression.

Keywords: poxvirus, vaccinia virus, monkeypox, resveratrol, DNA synthesis, gene expression, antiviral

\section{INTRODUCTION}

Smallpox is a deadly disease, responsible for approximately 300 million human deaths in the 20th century alone. Smallpox is caused by the variola virus, the most notorious member of the family Poxviridae (Miller et al., 2001). Despite the eradication of smallpox 37 years ago, poxviruses are of renewed interest due to their continuous impact on public health. Specifically, many poxviruses cause other human and animal diseases. For example, monkeypox, a zoonotic disease endemic in Central and Western Africa, caused an outbreak in humans in the United States (US) in 2003 (Reed et al., 2004; Bayer-Garner, 2005). Molluscum contagiosum accounts for 1 in 500 outpatient visits per year in the United States (Reynolds et al., 2009). Additionally, there is a concern that variola virus, the causative agent of smallpox, can potentially be used as a biological weapon from unsecured stocks or genetic engineering. Humans are particularly vulnerable to smallpox in the post-smallpox immunization era due to the absence of routine vaccination, waning immunity, and lower proportion of vaccinated individuals in the current population. In fact, between 1980 and 2010, the monkeypox incidence in Central Africa has increased 20 times after the discontinuation of smallpox immunization (Rimoin et al., 2010). In addition, poxviruses are developed as vectors for vaccine development against infectious diseases and as anti-cancer agents (Rerks-Ngarm et al., 2009; Draper and Heeney, 2010; Breitbach et al., 2011; Altenburg et al., 2014; Izzi et al., 2014). There are no FDA-approved drugs for poxvirus-infection treatment. Cidofovir, a drug for human cytomegalovirus infection, is an off-label drug to treat poxvirus infection (Robbins et al., 2005; 
Lu et al., 2011; Dower et al., 2012). There were also a number of small-molecule inhibitors of poxviruses identified in the past years, for example, CMX001, Tecovirimat (ST-246), and CMLDBU6128 (Quenelle et al., 2007; Huggins et al., 2009; Jordan et al., 2009). However, resistant viruses to the compounds were isolated in cell culture, including CMX001 and ST-246 (Yang et al., 2005; Andrei et al., 2006; Farlow et al., 2010). A combination therapy may be required to treat infected individuals, which demands the identification and characterization of additional poxvirus inhibitors.

Resveratrol is a natural polyphenol stilbenoid found in grapes, berries, and a number of other plants. Extensive studies have been carried out to investigate its functions in modulating lifespan, metabolism, cancer, and other diseases (Fremont, 2000). Resveratrol inhibits replication of a number of viruses, such as influenza virus, herpes simplex virus, enterovirus, hepatitis $C$ virus, respiratory syncytial virus, human immunodeficiency virus, varicella zoster virus, Epstein-Barr virus, African swine fever virus, and duck enteritis virus (Docherty et al., 1999, 2006; Palamara et al., 2005; Nakamura et al., 2010; Galindo et al., 2011; Espinoza et al., 2012; Xie et al., 2012; Xu et al., 2013; Abba et al., 2015; Zhang et al., 2015). The antiviral mechanisms of resveratrol against these viral infections are diverse and include inhibition of viral protein synthesis, DNA synthesis, and modulation of host functions important for viral infection (Abba et al., 2015). In contrast to the above-mentioned viruses, resveratrol facilitates Kaposi's-sarcoma associated herpesvirus (KSHV) reactivation from latency in several cell lines through enhancing mitochondrial function of infected cells (Yogev et al., 2014). Nevertheless, the effect of resveratrol on poxvirus replication has not been examined. A previous study showed that several polyphenols, including resveratrol, directly bind to and may inhibit vaccinia virus (VACV, the prototypic member of poxviruses)-encoded N1 protein, a cellular apoptotic regulator (Cheltsov et al., 2010). However, N1L is a non-essential gene and deletion of N1L from VACV genome does not affect VACV infection in cultured cells (Bartlett et al., 2002). Therefore, it is unlikely that resveratrol can prevent VACV infection through N1 protein in cell culture.

Here, we demonstrated that resveratrol could strongly suppress VACV replication in multiple cell types. We also showed that resveratrol directly targeted VACV DNA synthesis step and the suppression was independent of the viral N1 protein. Resveratrol also suppressed monkeypox virus (MPXV) replication.

\section{MATERIALS AND METHODS}

\section{Cell Culture}

BS-C-1 cells (ATCC-CCL26) were cultured in Eagle's Minimum Essential Medium (EMEM). HeLa cells (ATCC-CCL2) were cultured in Dulbecco's Modified Eagle Medium (DMEM). Normal human dermal fibroblasts (NHDFs, ATCC PCS-201-010) and human foreskin fibroblasts (HFFs, kindly provided by Dr. Bernard Moss) were also cultured in DMEM. The EMEM and DMEM were supplemented with $10 \%$ fetal bovine serum (FBS),
L-glutamine $(2 \mathrm{mM})$, streptomycin $(100 \mu \mathrm{g} / \mathrm{mL})$, and penicillin (100 units $/ \mathrm{mL}$ ). Cells were cultured in an incubator with $5 \% \mathrm{CO}_{2}$ at $37^{\circ} \mathrm{C}$.

\section{Cell Viability Assay and Calculation of $50 \%$ Cytotoxicity Concentration $\left(\mathrm{CC}_{50}\right)$}

HeLa cells and HFFs were cultured in 12-well plates. The cells were treated with DMSO or resveratrol at a series of concentrations. Cell viability was measured using trypanblue exclusion test (Strober, 2015). After $24 \mathrm{~h}$ of treatment, cells in each well were treated with $300 \mu \mathrm{L}$ of trypsin and resuspended with $500 \mu \mathrm{L}$ of DMEM by pipetting. Twenty microliters of cell suspension was gently mixed with $20 \mu \mathrm{L}$ of $4 \%$ trypan blue. The numbers of cells were measured with a hemocytometer. The $\mathrm{CC}_{50}$ was calculated using relative cell viability at different resveratrol concentrations by linear regression analysis.

\section{Viruses, Viral Infection, and Titration}

Vaccinia virus Western Reserve (WR, ATCC VR-1354) strain was amplified and purified as described previously (Earl et al., 2001a). Recombinant N1L-deleted VACV was generated by homologous recombination and the N1L gene was replaced with a green fluorescent protein (GFP) gene. Briefly, PCR product of GFP coding sequence under a late P11 promoter flanked by 500bp homologous sequences upstream and downstream N1L gene was transfected into VACV-infected HeLa cells. The transfected cells were collected at $24 \mathrm{~h}$ post-infection (hpi). Recombinant viruses expressing GFP were clonally purified by multiple rounds of plaque isolation (Earl et al., 2001b). Recombinant VACV with the correct insertion or deletion was verified by PCR. The recombinant VACV that expresses GFP under a synthetic early/late VACV promoter (Chakrabarti et al., 1997) and dsRED under P11 VACV promoter was generated using a similar procedure. Recombinant virus vP11-Fluc that expresses firefly luciferase gene under the late VACV P11 promoter was described elsewhere (Bengali et al., 2011). MPXV MPXV-WA 2003-044 and MPXV-ROC 2003-358 clades were utilized in this study. Preparation, infection, and titration of VACV and MPXV were carried out as described previously (Earl et al., 2001a). For infection, cells were incubated with desired amount of viruses in DMEM (containing 2.5\% FBS). After $1 \mathrm{~h}$ of incubation at $37^{\circ} \mathrm{C}$ in $5 \% \mathrm{CO}_{2}$, virus-containing DMEM was replaced with fresh DMEM (containing 2.5\% FBS) and further incubated for desired amount of time. For titration, BS-C-1 cells cultured in 6- or 12-well plates were infected with serial diluted viral samples and incubated in DMEM (containing 2.5\% FBS and 0.5\% methyl cellulose) for $48 \mathrm{~h}$. The cells were stained with $0.1 \%$ crystal violet for $5 \mathrm{~min}$ and washed with water before counting the number of plaques.

\section{Measurement and Calculation of $50 \%$ Inhibiting Concentration $\left(\mathrm{IC}_{50}\right)$}

HeLa cells or HFFs were cultured in 12-well plates. The cells were infected with VACV at a multiplicity of infection (MOI) of 1 in the presence of DMSO or resveratrol at a series of 
concentrations. After $24 \mathrm{hpi}$, virus titers were measured by a plaque assay. The $\mathrm{IC}_{50}$ was calculated using virus inhibitory efficiency at different resveratrol concentrations by linear regression analysis.

\section{Antibodies and Chemical Inhibitors}

Antibodies against VACV L2 protein, P4a (A10) protein, and whole VACV viral particle were kindly provided by Dr. Bernard Moss. Antibody against human GAPDH was purchased from Abcam (Cambridge, MA, United States). Chemicals cytosine-1- $\beta$-D-arabinofuranoside (AraC), resveratrol, and hydroxyurea were purchased from Sigma (St. Louis, MO, United States).

\section{Western Blotting Analysis}

Cells were collected and lysed in NP-40 cell lysis buffer $(150 \mathrm{mM}$ $\mathrm{NaCl}, 1 \% \mathrm{NP}-40,50 \mathrm{mM}$ Tris-Cl, pH 8.0). Cell lysates were reduced by $100 \mathrm{mM}$ DTT and denatured by sodium dodecyl sulfate-polyacrylamide gel electrophoresis (SDS-PAGE) loading buffer and boiling for $3 \mathrm{~min}$ before SDS-PAGE, followed by transferring to a polyvinylidene difluoride membrane. The membrane was then blocked in TBS-Tween (TBST) $[50 \mathrm{mM}$ Tris- $\mathrm{HCl}$ ( $\mathrm{pH} 7.5$ ), $200 \mathrm{mM} \mathrm{NaCl}, 0.05 \%$ Tween 20] containing $5 \%$ skim milk and $1 \%$ bovine serum albumin for $1 \mathrm{~h}$, incubated with primary antibody in the same TBST-milk buffer for $1 \mathrm{~h}$, washed with TBST three times for 10 min each time, incubated with horseradish peroxidase-conjugated secondary antibody for $1 \mathrm{~h}$, washed three times with TBST, and developed with chemiluminescent substrate (National Diagnostics, Atlanta, GA, United States). The whole procedure was carried out at room temperature. Antibodies were stripped from the membrane by Restore (Thermo Fisher Scientific, Waltham, MA, United States) for western blot analysis using another antibody.

\section{Luciferase Assay}

Firefly luciferase activities were measured by an ENSPIRE plate reader (PerkinElmer, Waltham, MA, United States) using the Luciferase Assay System (Promega, Madison, WI, United States) according to manufacturer's instructions.

\section{Plasmid Replication in VACV-Infected Cells}

Total DNA was isolated using E.Z.N.A. ${ }^{\circledR}$ Blood DNA Kit (Omega Bio-Tek, Inc., Norcross, GA, United States). One microgram of DNA was treated with a DpnI enzyme to digest originally transfected input plasmid DNA (amplified from Escherichia coli, with methylation on DpnI recognition site) but not the plasmid DNA amplified in mammalian cells (no methylation in DpnI site). The plasmid DNA amounts were then measured using qPCR using a pair of primers that amplify a fragment containing a DpnI site.

\section{Quantitative Real-Time PCR}

Total DNA was extracted from mock- or VACV-infected cells at indicated time points using E.Z.N.A. ${ }^{\circledR}$ Blood DNA Kit. Relative viral DNA levels were quantified by CFX96 real-time PCR instrument (Bio-Rad, Hercules, CA, United States) with All-inone $^{\mathrm{TM}} 2 \times$ qPCR mix (GeneCopoeia) and primers specific for VACV and human genomes, respectively. The qPCR program was started with initial denaturation step at $95^{\circ} \mathrm{C}$ for $3 \mathrm{~min}$, followed by 40 cycles of denaturation at $95^{\circ} \mathrm{C}$ for $10 \mathrm{~s}$, annealing and reading fluorescence at $52^{\circ} \mathrm{C}$ for $30 \mathrm{~s}$, and extension at $72^{\circ} \mathrm{C}$ for $30 \mathrm{~s}$. The primers used in this study are:

\section{C11pF: AAACACACACTGAGAAACAGCATAAA; C11pR: ACTATCGGCGAATGATCTGATTATC;} GAPDH-F: ACATCAAGAAGGTGGTGAAGCA; GAPDH-R: CTTGACAAAGTGGTCGTTGAGG. The primers used for recombinant N1L-deletion VACV characterization are:

N1-F: TTATTTTTCACCATATAGATCAATCATTAGA TCAT.

N1-R: ATGAGGACTCTACTTATTAGATATATTCTTT GGAG.

Puc19-F: TGCGCGTAATCTGCTGCTTG. Puc19-R: CGAGGTATGTAGGCGGTGCT.

\section{Statistical Analysis}

All titration data were represented as the means of at least three independent experiments. One-tailed paired $T$-test was used to access for significant difference between two means with $P<0.05$.

\section{RESULTS}

\section{Resveratrol Suppresses VACV Replication in Immortal and Primary Human Cells}

To test the effect of resveratrol on the viability, HeLa cells, an immortal cervical cancer cell line (Scherer et al., 1953), were treated at a series of concentrations. Cell viability assay showed that resveratrol caused $50 \% \mathrm{HeLa}$ cell death $\left(\mathrm{CC}_{50}\right)$ at the concentrations of $157.75 \mu \mathrm{M}$ in $24 \mathrm{~h}$ (Figure 1A and Table 1). Consistent with the result, no significant morphological change was observed for HeLa cells at the concentration of $50 \mu \mathrm{M}$ (Figure 1B). We then examined the effect of resveratrol on VACV replication. HeLa cells were infected with VACV at an MOI of 1 in the presence of a series of concentrations of resveratrol and the viral titers were measured $24 \mathrm{hpi}$. The concentration of resveratrol that resulted in $50 \%$ inhibition $\left(\mathrm{IC}_{50}\right)$ of VACV replication was $4.72 \mu \mathrm{M}$ (Figure $\mathbf{1 C}$ and Table $\mathbf{1}$ ). Resveratrol reduced virus yield by more than 120 -fold at the concentration of $50 \mu \mathrm{M}$ (Figure 1C). The inhibitory effect of VACV replication by resveratrol is comparable to a wellcharacterized VACV inhibitor, hydroxyurea, which is known to prevent VACV DNA synthesis and decreased virus yield by approximately 200 -fold at the concentration of $10 \mathrm{mM}$ under the same infection conditions (Figure 1D). We examined the effect of resveratrol on multiple rounds of VACV replication by infecting HeLa cells at a low MOI of 0.01 and measuring the viral yield at different times post VACV infection. We observed significant reduction of viral titers in resveratrol-treated cells started from 8 hpi (Figure 1E), again demonstrating that resveratrol severely 


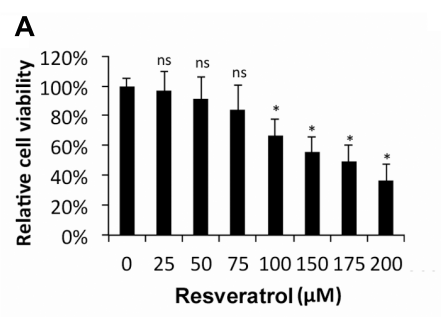

B

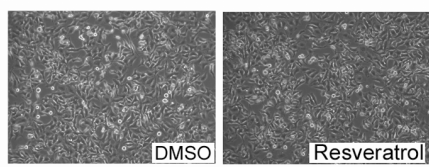

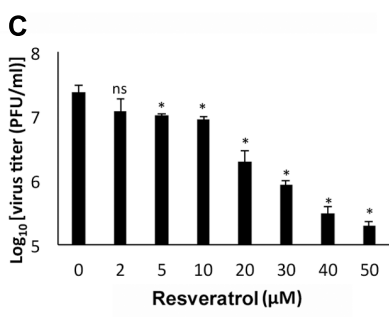

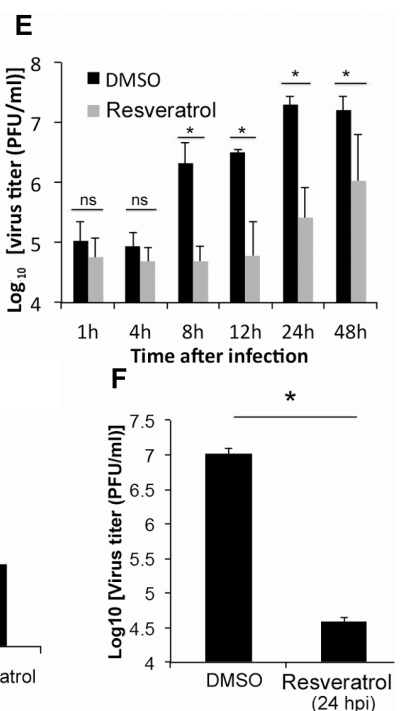

FIGURE 1 | Resveratrol suppresses VACV replication in HeLa cells. (A) HeLa cells were treated with DMSO or resveratrol at the indicated concentrations for $24 \mathrm{~h}$. Cell viability was measured using trypan blue exclusion test. (B) HeLa cells were imaged under the bright field of a microscope after DMSO or resveratrol (50 $\mu \mathrm{M}$ ) treatment for 24 h. (C) HeLa cells were infected with VACV at an MOI of 1 in the presence of resveratrol at the indicated concentrations. Virus yield at 24 hpi were determined by a plaque assay. (D) HeLa cells were infected with VACV at an MOI of 1 in the presence of DMSO, hydroxyurea (10 mM), or resveratrol (50 $\mu \mathrm{M})$. Virus yield at 24 hpi were determined by a plaque assay. (E) HeLa cells were infected with VACV at an MOI of 0.01 in the presence of DMSO or $50 \mu M$ resveratrol. Virus titers were determined by a plaque assay at the indicated time points. (F) HeLa cells were infected with VACV at an MOI of 0.001 treated with DMSO or $50 \mu \mathrm{M}$ resveratrol at $24 \mathrm{hpi}$. Virus titers were determined by a plaque assay at $72 \mathrm{hpi}$. The asterisk indicates significant difference $(P<0.05)$ and the ns indicates no significant difference between DMSO-treated cells and resveratrol- or hydroxyurea-treated cells. The error bar indicates standard deviation.

impaired the replication of VACV in HeLa cells. Moreover, the addition of resveratrol at 24 hpi still reduced VACV replication by 250 -fold when the initial MOI is low (0.001) (Figure 1F), suggesting a possible use of resveratrol to prevent viral spreading post infection.

The effect of resveratrol on VACV replication in primary human cells such as HFFs was also tested. The $\mathrm{CC}_{50}$ concentration of resveratrol on HFF was $176.88 \mu \mathrm{M}$ (Figure 2A and Table 1). In fact, at the concentration of up to $100 \mu \mathrm{M}$, resveratrol did not affect the morphology of HFFs (Figure 2B). The $\mathrm{IC}_{50}$ concentration of resveratrol in HFFs was $3.51 \mu \mathrm{M}$ and the virus yield of VACV from $50 \mu \mathrm{M}$ resveratrol-treated HFFs was reduced by approximately 200-fold at an MOI of 1 (Figure 2C and Table 1). Moreover, treatment of HFFs with $100 \mu \mathrm{M}$ of resveratrol protected the HFFs from VACV infectioninduced cytopathic effects of the cells (Figure 2D). In addition, resveratrol also reduced the replication of VACV in another primary human cell type, NHDF (not shown). Taken together, our results demonstrate that resveratrol dramatically reduces VACV replication in different human cell types.

\section{Resveratrol Suppresses MPXV Replication}

We examined the effect of resveratrol on MPXV replication. HeLa cells were infected with MPXV-WA and MPXV-ROC, respectively, at an MOI of 1 in the presence of a series of concentrations of resveratrol and the viral titers were measured 24 hpi. As shown in Figures 3A,B, $50 \mu \mathrm{M}$ resveratrol reduced the virus yield of MPXV-WA and MPXV-ROC clades by 195- and 38 -fold, respectively. The $\mathrm{IC}_{50}$ was $12.41 \mu \mathrm{M}$ for WA strain and $15.23 \mu \mathrm{M}$ for ROC strain (Table 2). The inhibitory effect of MPXV replication by resveratrol was comparable to the wellcharacterized orthopoxvirus (OPXV) inhibitor, AraC, in the corresponding parallel experiments (Figure 3).

\section{Resveratrol Suppresses N1L-Deleted VACV Replication}

$\mathrm{N} 1 \mathrm{~L}$ encodes a viral virulence factor that is expressed at early stage of VACV gene expression and regulates host cell apoptosis (Bartlett et al., 2002; Yang et al., 2010). It has been reported that some polyphenols, including resveratrol, could directly bind to and may inhibit the function of N1 protein (Cheltsov et al., 2010). The authors further speculated that resveratrol might inhibit VACV replication by targeting the N1 protein. However, the effect of resveratrol on VACV replication was not tested in the aforementioned study. Moreover, the N1L is not an essential VACV gene and the deletion of N1L from VACV genome was

TABLE 1 | Inhibitory effect of resveratrol on VACV replication and cytotoxicity.

\begin{tabular}{lcc}
\hline Cells & $\mathbf{I C}_{\mathbf{5 0}}(\boldsymbol{\mu} \mathbf{M})^{\mathbf{a}}$ & $\mathbf{C C}_{\mathbf{5 0}}(\boldsymbol{\mu} \mathbf{M})^{\mathbf{b}}$ \\
\hline HFF & $3.51 \pm 1.22$ & $176.88 \pm 17.44$ \\
HeLa & $4.72 \pm 2.34$ & $157.75 \pm 23.66$
\end{tabular}

aThe concentration of resveratrol that reduces the yield of VACV by $50 \%$. 'b The concentration of resveratrol that causes $50 \%$ cell death. 


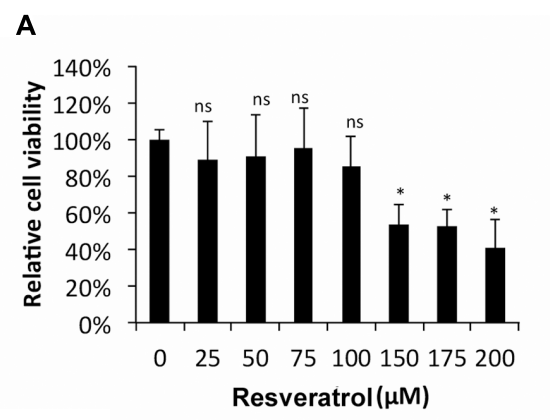

B

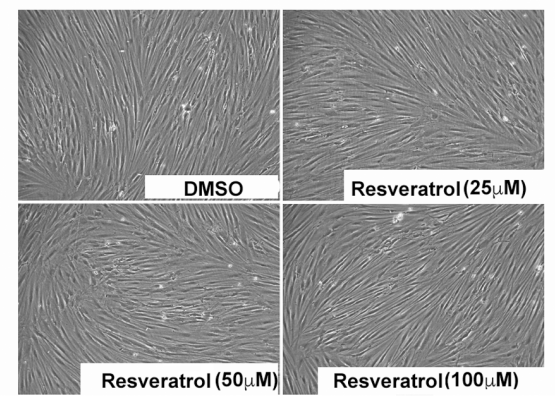

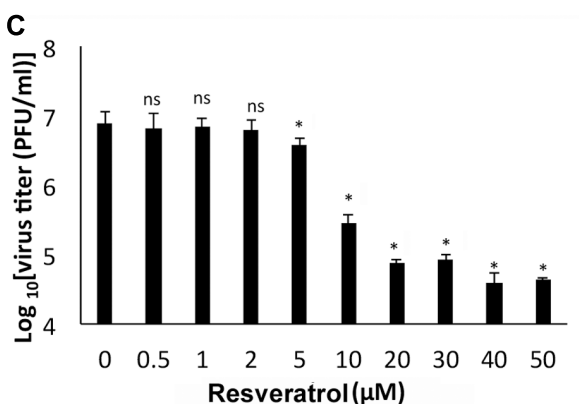

D

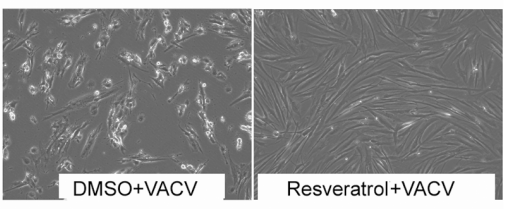

FIGURE 2 | Resveratrol suppresses VACV replication in HFFs. (A) HFFs were treated with DMSO or resveratrol at the indicated concentrations for 24 h. Cell viability was measured using trypan blue exclusion test. (B) DMSO- or resveratrol-treated cells were imaged under the bright field of a microscope. (C) HFFs were infected with VACV at an MOI of 1 in the presence of resveratrol at the indicated concentrations. Virus yield at 24 hpi was determined by a plaque assay. (D) HFFs were infected with VACV at an $\mathrm{MOI}$ of 0.01 in the presence of resveratrol at the indicated concentrations. At 72 hpi, cells were imaged under the bright field of a microscope. The asterisk indicates significant difference $(P<0.05)$ and the ns indicates no significant difference between DMSO-treated cells and resveratrol-treated cells. The error bar indicates standard deviation.

not shown to affect VACV replication in cultured cells (Bartlett et al., 2002). Based on these facts, we reasoned that prevention of VACV replication by resveratrol is not through the N1 protein. To test it, we replaced the N1L gene with a GFP gene in the VACV genome through homologous recombination (Figure 4A). Consistent with a previous observation (Bartlett et al., 2002), the deletion of N1L did not affect VACV replication and viral yields (Figure 4B). As expected, resveratrol similarly suppressed VACVDel-N1L virus (Figure 4C), indicating that inhibitory effect is not mediated through the N1 protein.

\section{Resveratrol Suppresses VACV Late, But Not Early Gene Expression}

To investigate the stage of viral life cycle targeted by resveratrol, we examined the effect of resveratrol treatment on VACV protein expression by Western blot analysis (Figure 5A). Anti-VACV serum was derived from rabbits immunized with purified VACV particles that comprise mostly viral structural proteins expressed at the late stage of VACV gene expression. P4a is a major viral core protein encoded by the VACV late gene A10L (Yang et al., 2011). L2 protein is involved in VACV morphogenesis and is expressed at the early stage of VACV gene expression (Yang et al., 2010; Maruri-Avidal et al., 2011a,b). DNA synthesis inhibitor AraC was used as a positive control. Western blots with anti-VACV serum and P4a antibodies demonstrated dramatic reduction in protein levels in the presence of resveratrol and at levels comparable to the AraC treatment. In contrast, both resveratrol and AraC treatments did not affect the expression level of the viral early protein L2 (Figure 5A). We also used a recombinant VACV that expressed GFP under an early/late VACV promoter and dsRED under a late VACV promoter to confirm suppression of late protein synthesis by resveratrol. HeLa cells infected with recombinant VACV expressing fluorescent proteins at an MOI of 1 in the presence of resveratrol, AraC, or vehicle control DMSO were observed under a fluorescent microscope. The results clearly showed that both resveratrol and AraC completely blocked dsRED expression that was expressed at the late stage of gene expression, while they only partially suppressed GFP expression at similar levels that could also be expressed at the early stage of VACV replication (Figure 5B). These results indicate that resveratrol has little or only moderate effect on VACV replication prior to viral early gene expression but affects a replication step between the early and late stages of gene expression.

The third approach we employed to examine the effect of resveratrol on VACV late gene expression was using a combination of hydroxyurea and resveratrol. Hydroxyurea blocks VACV DNA synthesis but not early gene expression (Katz et al., 1974). In the control experiment, hydroxyurea and resveratrol were confirmed for their inhibitory effects on expression of VACV late promoter-controlled firefly luciferase gene of vP11-Fluc in HeLa cells (Figure 5C). In the parallel experiment, HeLa cells were infected with vP11-Fluc for $3 \mathrm{~h}$ in the 

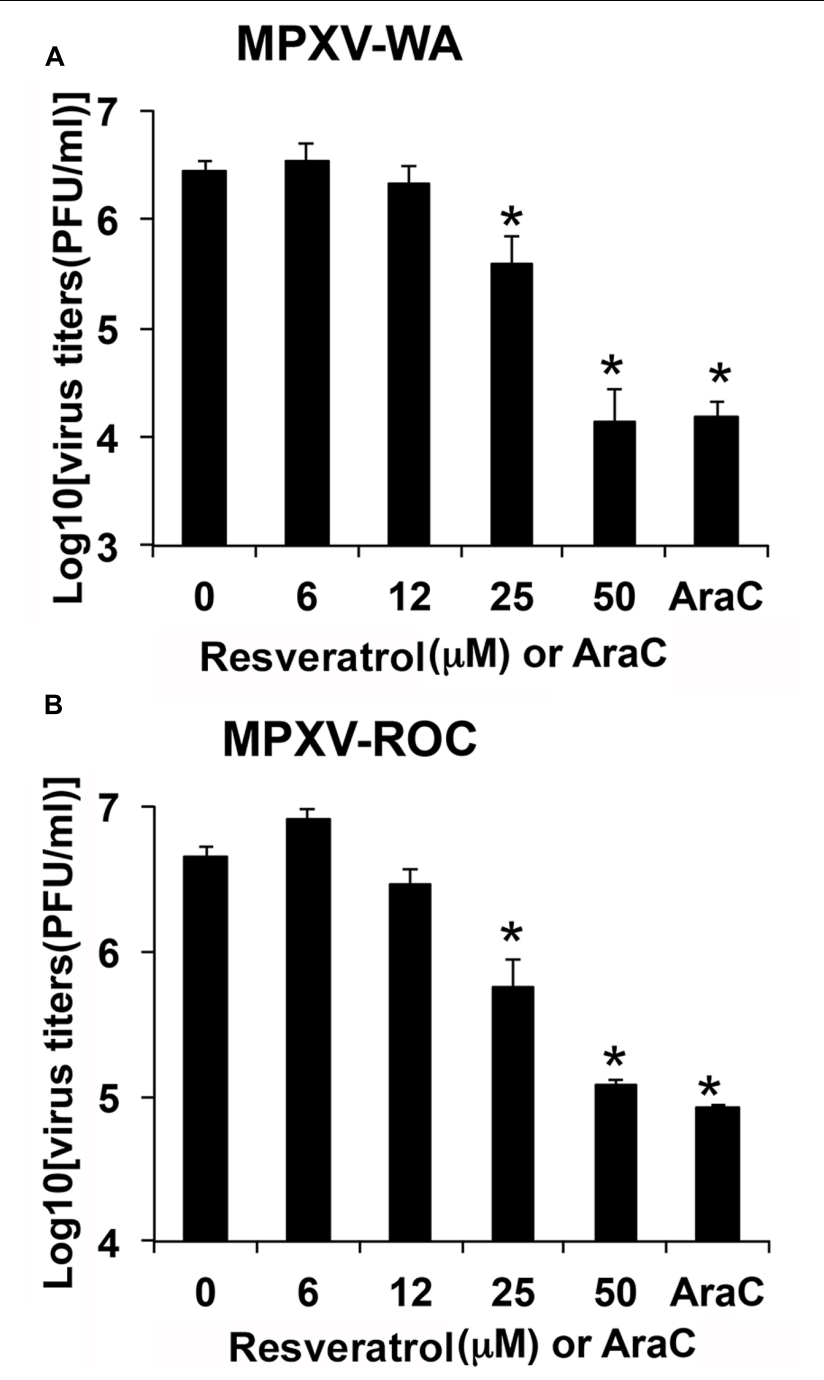

FIGURE 3 | Resveratrol suppresses monkeypox infection. HeLa cells were infected with MPXV-WA (A) or MPXV-ROC (B) at an MOI of 1 in the presence of resveratrol at the indicated concentrations or $\operatorname{AraC}(40 \mu \mathrm{g} / \mathrm{mL})$. Virus yield at $24 \mathrm{hpi}$ was determined by a plaque assay. The asterisk indicates significant difference $(P<0.05)$ between DMSO-treated cells and resveratrol- or AraC-treated cells. The error bar indicates standard deviation.

presence of hydroxyurea, which allowed early gene expression. The hydroxyurea was washed away and DMSO or resveratrol was added and incubated for an additional $3 \mathrm{~h}$. As can be seen, resveratrol still reduced luciferase activity while DMSO could not (Figure 5C).

Together, these results indicated that resveratrol affected a post-replication step after VACV early gene expression.

\section{Resveratrol Interferes VACV DNA Synthesis}

The effect of resveratrol on VACV DNA synthesis was investigated since it is essential for post-replicative gene expression (intermediate and late protein synthesis). VACV DNA synthesis starts between 2 and 4 hpi in infected HeLa cells under the conditions used in this study (Yang et al., 2010). We examined VACV DNA amounts in VACV-infected HeLa cells at 1 and 24 hpi using quantitative real-time PCR (AraC was used as positive control). Our results indicated that resveratrol treatment significantly reduced VACV DNA amount at 24 hpi (Figure 6A). The VACV DNA was 237-fold higher in DMSO-treated cells, while the viral DNA amounts only increased 35- and 6-fold in resveratrol- and AraC-treated cells, respectively (Figure 6A).

We tested the direct inhibitory effect on DNA synthesis by resveratrol through examining plasmid DNA synthesis in VACVinfected cells as circular DNA can be replicated in VACV-infected cells that require all known viral proteins needed for VACV DNA synthesis (DeLange and McFadden, 1986; De Silva and Moss, 2005). We transfected pUC19 plasmid into HeLa cells for $12 \mathrm{~h}$ and then infected with VACV or mock-infected in the presence or absence of resveratrol and AraC. Total DNA was isolated from cells at 24 hpi and treated with DpnI that only digests methylated input DNA. The DNA was then measured using specific primers amplifying a pUC19 fragment containing the DpnI digestion site. Dpn-resistant plasmid DNA increased 12-fold in VACVinfected cells compared to DMSO treatment. However, there was only a 2- to 3-fold increase of DpnI-resistant plasmid DNA in VACV-infected cells treated with resveratrol or AraC treatment (Figure 6B). This result indicated that resveratrol could interfere viral DNA synthesis directly in VACV-infected cells.

\section{DISCUSSION}

Our study, for the first time, demonstrated a strong suppressive effect of resveratrol on poxvirus replication. Similar to other viruses, VACV replication is generally divided into entry, gene expression, genome replication, viral particle assembly, and exit steps. VACV gene expression is programmed as a cascade to express viral genes at early, intermediate, and late stages (Moss, 2013a). The early gene expression starts immediately after VACV enters into the infected cells, as the viral infectious particles package all the factors and enzymes needed for early viral mRNA synthesis. The viral early gene products include those necessary factors for viral DNA synthesis. The VACV DNA synthesis is required for viral intermediate, and subsequently, late gene expression. The intermediate and late gene products comprise most of the structural proteins to build infectious viral particles (Moss, 2013a). Our study indicates that the resveratrol directly targets viral DNA synthesis step to prevent VACV replication. Genome uncoating is a step needed to expose encapsidated viral DNA as a template for DNA synthesis. Because resveratrol does not block synthesis of viral early proteins and the viral genome uncoating factor D5 is an early protein (Kilcher et al., 2014),

TABLE 2 | Inhibitory effect of resveratrol on MPXV replication in HeLa cells.

\begin{tabular}{lr}
\hline MPXV strains & $\mathbf{I C}_{\mathbf{5 0}}(\boldsymbol{\mu} \mathbf{M})^{\mathbf{a}}$ \\
\hline WA & $12.41 \pm 3.28$ \\
ROC & $15.23 \pm 2.71$ \\
\hline
\end{tabular}

a The concentration of resveratrol that reduces the yield of MPXV by $50 \%$. 

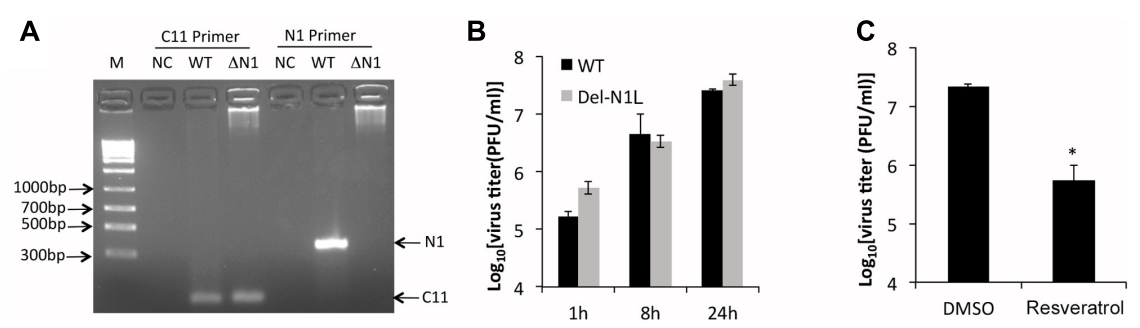

FIGURE 4 | Resveratrol suppresses N1L-deleted VACV replication. (A) Deletion of N1L gene from recombinant virus was confirmed by PCR with two pairs of primer specific for C11R gene (positive control) and N1L gene, respectively. (B) HeLa cells were infected with wild type and N1L-deleted VACV at an MOI of 1. Virus growth was determined by a plaque assay. (C) HeLa cells were infected with N1L-deleted VACV at an MOI of 1 in the presence or absence of resveratrol (50 $\mu$ M). Virus titers were determined by a plaque assay at 24 hpi. The asterisk indicates significant difference $(P<0.05)$. The error bar indicates standard deviation.
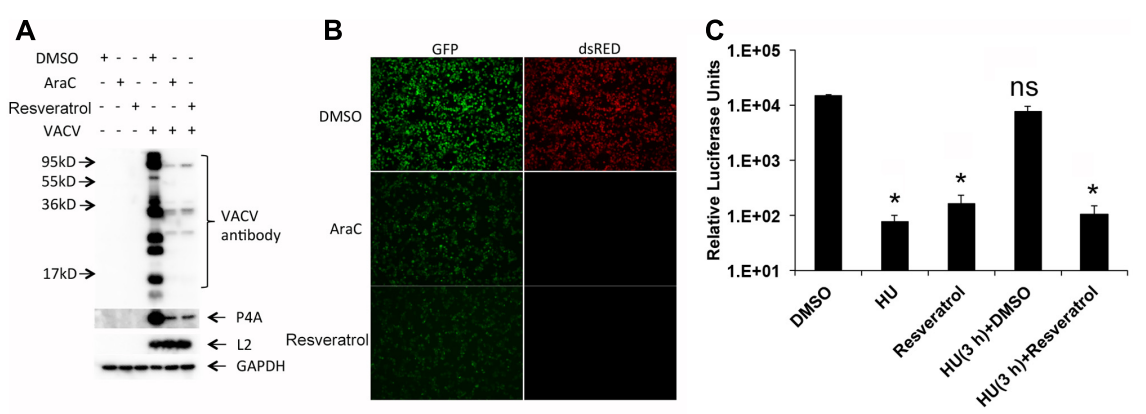

FIGURE 5 | Resveratrol suppresses VACV late, but not early gene expression. (A) HeLa cells were infected with VACV at an MOI of 1 in the presence of DMSO, AraC $(40 \mu \mathrm{g} / \mathrm{mL})$, or resveratrol $(50 \mu \mathrm{M})$. At $24 \mathrm{hpi}$, the expression of viral proteins in infected cells was detected by western blotting using the indicated antibodies. (B) HeLa cells were infected with a recombinant VACV carrying a GFP gene under an early/late VACV promoter and a dsRED gene under a late VACV promoter at an $\mathrm{MOI}$ of 1 in the presence of DMSO, AraC $(40 \mu \mathrm{g} / \mathrm{mL})$, or resveratrol $(50 \mu \mathrm{M})$. The expression of GFP and dsRED was observed and imaged using a fluorescent microscope. (C) HeLa cells were infected with VACV-P11-Fluc at an MOI of 1 and treated with hydroxyurea (HU, $10 \mathrm{mM}$ ) from 0 to 3 hpi. Then hydroxyurea-containing medium was washed away and replaced with cell culture medium containing DMSO or resveratrol (50 $\mu \mathrm{M})$, and further incubated for another $3 \mathrm{~h}$ until luciferase activity in the infected cell lysates was measured. Luciferase activities from infected cells treated with only DMSO, hydroxyurea, or resveratrol through $0-6$ hpi were also measured. The asterisk indicates significant difference $(P<0.05)$ between control and treated cells. The ns indicates no significant difference. The error bar indicates standard deviation.
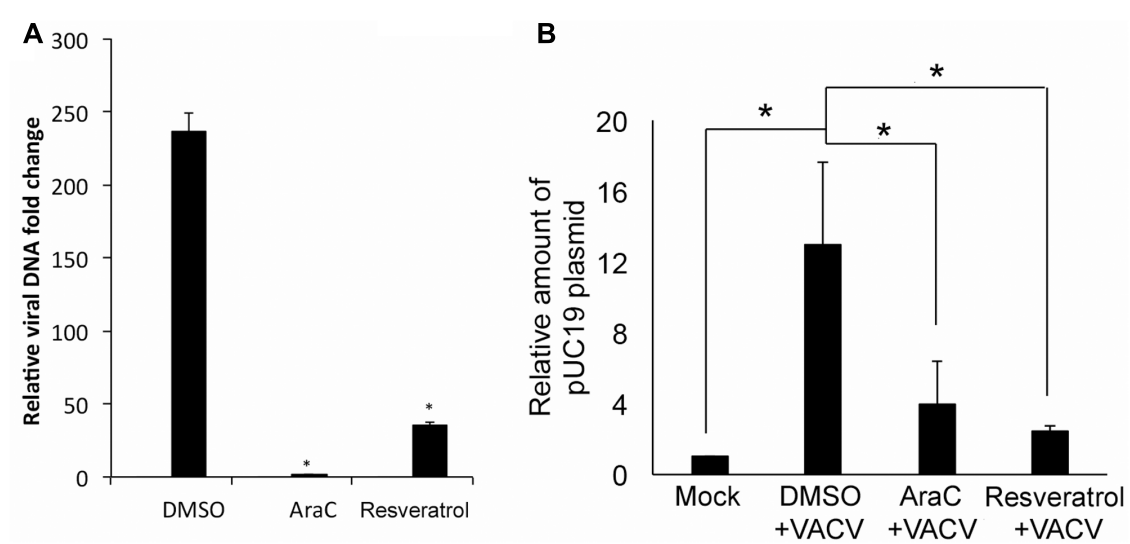

FIGURE 6 | Resveratrol suppresses VACV DNA synthesis. (A) HeLa cells were infected with VACV at an MOI of 1 in the presence of DMSO, AraC (40 $\mu \mathrm{g} / \mathrm{mL})$, or resveratrol $(50 \mu \mathrm{M})$. Relative viral DNA levels in infected cells were determined by real-time PCR at 1 and 24 hpi. The viral DNA level at 24 hpi was determined as the fold to the viral DNA level at $1 \mathrm{hpi}$. (B) HeLa cells were transfected with $200 \mathrm{ng}$ of pUC19 plasmid and incubated overnight. The cells were then infected with VACV at an $\mathrm{MOI}$ of 5 or mock-infected in the presence of AraC, resveratrol, or DMSO. Total DNA was extracted from the cells at 24 hpi and $1 \mu \mathrm{g}$ of total DNA was digested with Dpnl at $37^{\circ} \mathrm{C}$ for $2 \mathrm{~h}$ followed by real-time qPCR using primers amplifying pUC19 fragment containing Dpnl digestion site. The asterisk indicates significant difference $(P<0.05)$ and the ns indicates no significant difference between 1 and 24 hpi. The error bar indicates standard deviation. 
it is unlikely that resveratrol can prevent poxvirus genome uncoating. However, we do not completely rule out the possibility that resveratrol interferes poxvirus genome uncoating to some extent. Interestingly, the effect is independent of the non-essential viral N1L gene, albeit resveratrol has been suggested to be an inhibitor of the VACV N1 protein (Cheltsov et al., 2010).

As the prototypic member of poxvirus family, VACV has a linear, double-stranded DNA genome that replicates entirely in the cytoplasm (Moss, 2013a). The size of the genome is approximately $200 \mathrm{kbp}$. Although the molecular mechanism involved in VACV DNA synthesis is not fully understood, it is known that the VACV genome encodes most proteins required for replicating its DNA genome (Moss, 2013b). These proteins include a DNA polymerase encoded by E9L gene (Jones and Moss, 1984; Traktman et al., 1984), a helicase-primase encoded by D5R (Roseman and Hruby, 1987), a processivity factor encoded by A20R (McDonald et al., 1997), a uracil DNA glycosylase encoded by D4R (Upton et al., 1993), and a few other proteins bearing different roles in copying the viral DNA (Moss, 2013b). It has been shown that resveratrol inhibits multiple mammalian DNA polymerases including polymerase alpha through its 4hydroxystyryl moiety, subsequently suppressing active DNA synthesis (Locatelli et al., 2005). As VACV DNA polymerase has considerable similarity to human polymerase alpha (Wang et al., 1989), it is highly possible that resveratrol interferes with VACV DNA polymerase activity directly. Resveratrol also modulates numerous cellular functions (Fremont, 2000); therefore, it is possible that resveratrol affects a cellular function that is important for VACV genome replication. However, the role of cellular functions in VACV DNA synthesis is poorly understood; thus, it is difficult to have an educated prediction of a specific cellular function that may be involved in this process.

All steps of VACV replication, from viral entry and exit, may be targeted for antiviral drug development. For example, mitoxantrone blocks VACV replication by targeting the virion assembly step (Deng et al., 2007). However, the viral DNA synthesis is one of the major targets for anti-poxvirus drug development. Several compounds that are used to treat poxvirus infection target the viral DNA synthesis step. Cidofovir, an acyclic nucleoside that is approved to treat cytomegalovirus infection in AIDS patients also exhibits anti-poxvirus activity by targeting DNA synthesis (Andrei and Snoeck, 2010). The

\section{REFERENCES}

Abba, Y., Hassim, H., Hamzah, H., and Noordin, M. M. (2015). Antiviral activity of resveratrol against human and animal viruses. Adv. Virol. 2015:184241. doi: 10.1155/2015/184241

Altenburg, A. F., Kreijtz, J. H., de Vries, R. D., Song, F., Fux, R., Rimmelzwaan, G. F., et al. (2014). Modified Vaccinia virus ankara (MVA) as production platform for vaccines against influenza and other viral respiratory diseases. Viruses 6, 2735-2761. doi: 10.3390/v6072735

Andrei, G., Gammon, D. B., Fiten, P., De Clercq, E., Opdenakker, G., Snoeck, R., et al. (2006). Cidofovir resistance in Vaccinia virus is linked to diminished virulence in mice. J. Virol. 80, 9391-9401. doi: 10.1128/JVI.00605-06 widely used poxvirus inhibitors, AraC, hydroxyurea, and a recently identified inhibitor, CMX001, also target VACV DNA synthesis (Quenelle et al., 2007). The identification of resveratrol as a VACV DNA synthesis inhibitor may allow for developing alternative or compensative strategies to better manage current and re-emergent poxvirus infections and complications caused by poxviruses-based therapeutics.

\section{CONCLUSION}

We showed that resveratrol, a member of natural plant polyphenols that is under extensive investigation of its effects on many biological processes, dramatically reduced VACV and MPXV replication. The suppression appears to affect the viral DNA synthesis step. The results will prompt further investigation of its effect on other poxvirus replication steps as well as the mechanism to inhibit VACV replication.

\section{AUTHOR CONTRIBUTIONS}

ZY, PS, and SC contributed to the conception of the study. SC, SR, and AP performed the experiments. SC and SR analyzed the data. ZY, SC, and PS wrote the manuscript.

\section{FUNDING}

This work was supported, in part by grants from the National Institutes of Health (P20GM113117, project 3) to ZY. AP was also supported by the Johnson Cancer Research Center at Kansas States University.

\section{ACKNOWLEDGMENTS}

The authors wish to thank Dr. Bernard Moss at the NIH for providing VACV WR strain, cells, and reagents. The authors also wish to thank other members in the Yang laboratory for helpful discussion. The findings and conclusions in this report are those of the authors and do not necessarily represent the official position of the Centers for Disease Control and Prevention, Atlanta, GA, United States, and Kansas State University.

Andrei, G., and Snoeck, R. (2010). Cidofovir activity against poxvirus infections. Viruses 2, 2803-2830. doi: 10.3390/v2122803

Bartlett, N., Symons, J. A., Tscharke, D. C., and Smith, G. L. (2002). The Vaccinia virus N1L protein is an intracellular homodimer that promotes virulence. J. Gen. Virol. 83(Pt 8), 1965-1976. doi: 10.1099/0022-1317-83-8-1965

Bayer-Garner, I. B. (2005). Monkeypox virus: histologic, immunohistochemical and electron-microscopic findings. J. Cutan. Pathol. 32, 28-34. doi: 10.1111/j. 0303-6987.2005.00254.x

Bengali, Z., Satheshkumar, P. S., Yang, Z., Weisberg, A. S., Paran, N., and Moss, B. (2011). Drosophila S2 cells are non-permissive for Vaccinia virus DNA replication following entry via low $\mathrm{pH}$-dependent endocytosis and early transcription. PLOS ONE 6:e17248. doi: 10.1371/journal.pone.0017248 
Breitbach, C. J., Burke, J., Jonker, D., Stephenson, J., Haas, A. R., Chow, L. Q., et al. (2011). Intravenous delivery of a multi-mechanistic cancer-targeted oncolytic poxvirus in humans. Nature 477, 99-102. doi: 10.1038/nature10358

Chakrabarti, S., Sisler, J. R., and Moss, B. (1997). Compact, synthetic, Vaccinia virus early/late promoter for protein expression. Biotechniques 23, 1094-1097.

Cheltsov, A. V., Aoyagi, M., Aleshin, A., Yu, E. C., Gilliland, T., Zhai, D., et al. (2010). Vaccinia virus virulence factor N1L is a novel promising target for antiviral therapeutic intervention. J. Med. Chem. 53, 3899-3906. doi: 10.1021/ jm901446n

De Silva, F. S., and Moss, B. (2005). Origin-independent plasmid replication occurs in Vaccinia virus cytoplasmic factories and requires all five known poxvirus replication factors. Virol. J. 2:23.

DeLange, A. M., and McFadden, G. (1986). Sequence-nonspecific replication of transfected plasmid DNA in poxvirus-infected cells. Proc. Natl. Acad. Sci. USA. 83, 614-618. doi: 10.1073/pnas.83.3.614

Deng, L., Dai, P., Ciro, A., Smee, D. F., Djaballah, H., and Shuman, S. (2007). Identification of novel antipoxviral agents: mitoxantrone inhibits Vaccinia virus replication by blocking virion assembly. J. Virol. 81, 13392-13402. doi: 10.1128/ JVI.00770-07

Docherty, J. J., Fu, M. M., Stiffler, B. S., Limperos, R. J., Pokabla, C. M., and DeLucia, A. L. (1999). Resveratrol inhibition of herpes simplex virus replication. Antiviral Res. 43, 145-155. doi: 10.1016/S0166-3542(99)00042-X

Docherty, J. J., Sweet, T. J., Bailey, E., Faith, S. A., and Booth, T. (2006). Resveratrol inhibition of varicella-zoster virus replication in vitro. Antiviral Res. 72, 171-177. doi: 10.1016/j.antiviral.2006.07.004

Dower, K., Filone, C. M., Hodges, E. N., Bjornson, Z. B., Rubins, K. H., Brown, L. E., et al. (2012). Identification of a pyridopyrimidinone inhibitor of orthopoxviruses from a diversity-oriented synthesis library. J. Virol. 86, 2632-2640. doi: 10.1128/JVI.05416-11

Draper, S. J., and Heeney, J. L. (2010). Viruses as vaccine vectors for infectious diseases and cancer. Nat. Rev. Microbiol. 8, 62-73. doi: 10.1038/nrmicro2240

Earl, P. L., Cooper, N., Wyatt, L. S., Moss, B., and Carroll, M. W. (2001a). Preparation of cell cultures and Vaccinia virus stocks. Curr. Protoc. Mol. Biol. 16, 16.16.1-16.16.18.

Earl, P. L., Moss, B., Wyatt, L. S., and Carroll, M. W. (2001b). Generation of recombinant vaccinia viruses. Curr. Protoc. Mol. Biol. 43, 16.17.1-16.17.19.

Espinoza, J. L., Takami, A., Trung, L. Q., Kato, S., and Nakao, S. (2012). Resveratrol prevents EBV transformation and inhibits the outgrowth of EBV-immortalized human B cells. PLOS ONE 7:e51306. doi: 10.1371/journal.pone.0051306

Farlow, J., Ichou, M. A., Huggins, J., and Ibrahim, S. (2010). Comparative whole genome sequence analysis of wild-type and cidofovir-resistant monkeypoxvirus. Virol. J. 7:110. doi: 10.1186/1743-422X-7-110

Fremont, L. (2000). Biological effects of resveratrol. Life Sci. 66, 663-673. doi: 10.1016/S0024-3205(99)00410-5

Galindo, I., Hernaez, B., Berna, J., Fenoll, J., Cenis, J. L., Escribano, J. M., et al. (2011). Comparative inhibitory activity of the stilbenes resveratrol and oxyresveratrol on African swine fever virus replication. Antiviral Res. 91, 57-63. doi: 10.1016/j.antiviral.2011.04.013

Huggins, J., Goff, A., Hensley, L., Mucker, E., Shamblin, J., Wlazlowski, C., et al. (2009). Nonhuman primates are protected from smallpox virus or monkeypox virus challenges by the antiviral drug ST-246. Antimicrob. Agents Chemother. 53, 2620-2625. doi: 10.1128/AAC.00021-09

Izzi, V., Buler, M., Masuelli, L., Giganti, M. G., Modesti, A., and Bei, R. (2014). Poxvirus-based vaccines for cancer immunotherapy: new insights from combined cytokines/co-stimulatory molecules delivery and "uncommon" strains. Anticancer Agents Med. Chem. 14, 183-189. doi: 10.2174/18715206113136660376

Jones, E. V., and Moss, B. (1984). Mapping of the Vaccinia virus DNA polymerase gene by marker rescue and cell-free translation of selected RNA. J. Virol. 49, $72-77$.

Jordan, R., Goff, A., Frimm, A., Corrado, M. L., Hensley, L. E., Byrd, C. M., et al. (2009). ST-246 antiviral efficacy in a nonhuman primate monkeypox model: determination of the minimal effective dose and human dose justification. Antimicrob. Agents Chemother. 53, 1817-1822. doi: 10.1128/AAC.01596-08

Katz, E., Margalith, E., and Winer, B. (1974). Synthesis of Vaccinia virus polypeptides in the presence of hydroxyurea. Antimicrob. Agents Chemother. 6, 647-650. doi: 10.1128/AAC.6.5.647
Kilcher, S., Schmidt, F. I., Schneider, C., Kopf, M., Helenius, A., and Mercer, J. (2014). siRNA screen of early poxvirus genes identifies the AAA+ ATPase D5 as the virus genome-uncoating factor. Cell Host Microbe 15, 103-112. doi: 10.1016/j.chom.2013.12.008

Locatelli, G. A., Savio, M., Forti, L., Shevelev, I., Ramadan, K., Stivala, L. A., et al. (2005). Inhibition of mammalian DNA polymerases by resveratrol: mechanism and structural determinants. Biochem. J. 389(Pt 2), 259-268. doi: 10.1042/ BJ20050094

Lu, Y. C., Fan, H. C., Wang, C. C., and Cheng, S. N. (2011). Concomitant use of acyclovir and intravenous immunoglobulin rescues an immunocompromised child with disseminated varicella caused multiple organ failure. J. Pediatr. Hematol. Oncol. 33, e350-e351. doi: 10.1097/MPH.0b013e3181ec0efb

Maruri-Avidal, L., Domi, A., Weisberg, A. S., and Moss, B. (2011a). Participation of Vaccinia virus 12 protein in the formation of crescent membranes and immature virions. J. Virol. 85, 2504-2511. doi: 10.1128/JVI.02505-10

Maruri-Avidal, L., Weisberg, A. S., and Moss, B. (2011b). Vaccinia virus L2 protein associates with the endoplasmic reticulum near the growing edge of crescent precursors of immature virions and stabilizes a subset of viral membrane proteins. J. Virol. 85, 12431-12441. doi: 10.1128/JVI.05573-11

McDonald, W. F., Klemperer, N., and Traktman, P. (1997). Characterization of a processive form of the Vaccinia virus DNA polymerase. Virology 234, 168-175. doi: 10.1006/viro.1997.8639

Miller, J., Engelberg, S., and Broad, W. J. (2001). Germs: The Ultimate Weapon. New York, NY: Simon \& Schuster Ltd.

Moss, B. (2013a). "Poxviridae: the viruses and their replication," in Fields Virology, Vol. 2, eds D. M. Knipe and P. M. Howley (Philadelphia: PA: Wolters Kluwer/Lippincott Williams \& Wilkins), 2129-2159.

Moss, B. (2013b). Poxvirus DNA replication. Cold Spring Harb. Perspect. Biol. 5, a010199. doi: 10.1101/cshperspect.a010199

Nakamura, M., Saito, H., Ikeda, M., Hokari, R., Kato, N., Hibi, T., et al. (2010). An antioxidant resveratrol significantly enhanced replication of hepatitis $\mathrm{C}$ virus. World J. Gastroenterol. 16, 184-192. doi: 10.3748/wjg.v16.i2.184

Palamara, A. T., Nencioni, L., Aquilano, K., De Chiara, G., Hernandez, L., Cozzolino, F., et al. (2005). Inhibition of influenza A virus replication by resveratrol. J. Infect. Dis. 191, 1719-1729. doi: 10.1086/429694

Quenelle, D. C., Prichard, M. N., Keith, K. A., Hruby, D. E., Jordan, R., Painter, G. R., et al. (2007). Synergistic efficacy of the combination of ST246 with CMX001 against orthopoxviruses. Antimicrob. Agents Chemother. 51, 4118-4124. doi: 10.1128/AAC.00762-07

Reed, K. D., Melski, J. W., Graham, M. B., Regnery, R. L., Sotir, M. J., Wegner, M. V., et al. (2004). The detection of monkeypox in humans in the Western Hemisphere. N. Engl. J. Med. 350, 342-350. doi: 10.1056/NEJMoa032299

Rerks-Ngarm, S., Pitisuttithum, P., Nitayaphan, S., Kaewkungwal, J., Chiu, J., Paris, R., et al. (2009). Vaccination with ALVAC and AIDSVAX to prevent HIV-1 infection in Thailand. N. Engl. J. Med. 361, 2209-2220. doi: 10.1056/ NEJMoa0908492

Reynolds, M. G., Holman, R. C., Yorita Christensen, K. L., Cheek, J. E., and Damon, I. K. (2009). The Incidence of Molluscum contagiosum among American Indians and Alaska Natives. PLOS ONE 4:e5255. doi: 10.1371/journal.pone. 0005255

Rimoin, A. W., Mulembakani, P. M., Johnston, S. C., Lloyd Smith, J. O., Kisalu, N. K., Kinkela, T. L., et al. (2010). Major increase in human monkeypox incidence 30 years after smallpox vaccination campaigns cease in the Democratic Republic of Congo. Proc. Natl. Acad. Sci. U.S.A. 107, 16262-16267. doi: 10.1073/pnas.1005769107

Robbins, S. J., Jackson, R. J., Fenner, F., Beaton, S., Medveczky, J., Ramshaw, I. A., et al. (2005). The efficacy of cidofovir treatment of mice infected with ectromelia (mousepox) virus encoding interleukin-4. Antiviral Res. 66, 1-7. doi: 10.1016/j.antiviral.2004.12.003

Roseman, N. A., and Hruby, D. E. (1987). Nucleotide sequence and transcript organization of a region of the Vaccinia virus genome which encodes a constitutively expressed gene required for DNA replication. J. Virol. 61, 1398-1406.

Scherer, W. F., Syverton, J. T., and Gey, G. O. (1953). Studies on the propagation in vitro of poliomyelitis viruses. IV. Viral multiplication in a stable strain of human malignant epithelial cells (strain HeLa) derived from an epidermoid carcinoma of the cervix. J. Exp. Med. 97, 695-710. doi: 10.1084/jem.97.5.695 
Strober, W. (2015). Trypan blue exclusion test of cell viability. Curr. Protoc. Immunol. 111, A.3B.1-A.3B.3. doi: 10.1002/0471142735.ima03bs111

Traktman, P., Sridhar, P., Condit, R. C., and Roberts, B. E. (1984). Transcriptional mapping of the DNA polymerase gene of Vaccinia virus. J. Virol. 49, 125-131.

Upton, C., Stuart, D. T., and McFadden, G. (1993). Identification of a poxvirus gene encoding a uracil DNA glycosylase. Proc. Natl. Acad. Sci. U.S.A. 90, 4518-4522. doi: $10.1073 /$ pnas. 90.10 .4518

Wang, T. S., Wong, S. W., and Korn, D. (1989). Human DNA polymerase alpha: predicted functional domains and relationships with viral DNA polymerases. FASEB J. 3, 14-21.

Xie, X. H., Zang, N., Li, S. M., Wang, L. J., Deng, Y., He, Y., et al. (2012). Resveratrol Inhibits respiratory syncytial virus-induced IL-6 production, decreases viral replication, and downregulates TRIF expression in airway epithelial cells. Inflammation 35, 1392-1401. doi: 10.1007/s10753-012-9452-7

Xu, J., Yin, Z., Li, L., Cheng, A., Jia, R., Song, X., et al. (2013). Inhibitory effect of resveratrol against duck enteritis virus in vitro. PLOS ONE 8:e65213. doi: 10.1371/journal.pone.0065213

Yang, G., Pevear, D. C., Davies, M. H., Collett, M. S., Bailey, T., Rippen, S., et al. (2005). An orally bioavailable antipoxvirus compound (ST-246) inhibits extracellular virus formation and protects mice from lethal orthopoxvirus Challenge. J. Virol. 79, 13139-13149. doi: 10.1128/JVI.79.20.13139-13149.2005

Yang, Z., Bruno, D. P., Martens, C. A., Porcella, S. F., and Moss, B. (2010). Simultaneous high-resolution analysis of Vaccinia virus and host cell transcriptomes by deep RNA sequencing. Proc. Natl. Acad. Sci. USA. 107, 11513-11518. doi: 10.1073/pnas.1006594107
Yang, Z., Reynolds, S. E., Martens, C. A., Bruno, D. P., Porcella, S. F., and Moss, B. (2011). Expression profiling of the intermediate and late stages of poxvirus replication. J. Virol. 85, 9899-9908. doi: 10.1128/JVI.05446-11

Yogev, O., Lagos, D., Enver, T., and Boshoff, C. (2014). Kaposi's sarcoma herpesvirus microRNAs induce metabolic transformation of infected cells. PLOS Pathog. 10:e1004400. doi: 10.1371/journal.ppat. 1004400

Zhang, L., Li, Y., Gu, Z., Wang, Y., Shi, M., Ji, Y., et al. (2015). Resveratrol inhibits enterovirus 71 replication and pro-inflammatory cytokine secretion in rhabdosarcoma cells through blocking IKKs/NF-kappaB signaling pathway. PLOS ONE 10:e0116879. doi: 10.1371/journal.pone.0116879

Conflict of Interest Statement: The authors declare that the research was conducted in the absence of any commercial or financial relationships that could be construed as a potential conflict of interest.

The reviewer IAB and handling Editor declared their shared affiliation.

Copyright () 2017 Cao, Realegeno, Pant, Satheshkumar and Yang. This is an openaccess article distributed under the terms of the Creative Commons Attribution License (CC BY). The use, distribution or reproduction in other forums is permitted, provided the original author(s) or licensor are credited and that the original publication in this journal is cited, in accordance with accepted academic practice. No use, distribution or reproduction is permitted which does not comply with these terms. 